\title{
A General 2D Meshless Interpolating Boundary Node Method Based on the Parameter Space
}

\author{
Hongyin Yang, Hailin Lu, and Xuyong Chen \\ School of Resource and Civil Engineering, Wuhan Institute of Technology, Wuhan 430073, China \\ Correspondence should be addressed to Xuyong Chen; cxyl314kl@126.com
}

Received 25 May 2017; Revised 13 September 2017; Accepted 20 September 2017; Published 13 November 2017

Academic Editor: Ivano Benedetti

Copyright (c) 2017 Hongyin Yang et al. This is an open access article distributed under the Creative Commons Attribution License, which permits unrestricted use, distribution, and reproduction in any medium, provided the original work is properly cited.

\begin{abstract}
The presented study proposed an improved interpolating boundary node method (IIBNM) for 2D potential problems. The improved interpolating moving least-square (IIMLS) method was applied to construct the shape functions, of which the delta function properties and boundary conditions were directly implemented. In addition, any weight function used in the moving least-square (MLS) method was also applicable in the IIMLS method. Boundary cells were required in the computation of the boundary integrals, and additional discretization error was not avoided if traditional cells were used to approximate the geometry. The present study applied the parametric cells created in the parameter space to preserve the exact geometry, and the geometry was maintained due to the number of cells. Only the number of nodes on the boundary was required as additional information for boundary node construction. Most importantly, the IIMLS method can be applied in the parameter space to construct shape functions without the requirement of additional computations for the curve length.
\end{abstract}

\section{Introduction}

The mesh procedure in the finite element method (FEM) and boundary element method (BEM) [1-5] presents at least two problems: (i) mesh generation under certain conditions is still arduous, time-consuming, and fraught with pitfalls and (ii) the geometry of the model is approximated by elements and thereby results in additional discretization error. Meshless (mesh-free) methods have been developed in the past decades to reduce the required effort for mesh generation. Many meshless methods have been proposed thus far, including the smoothed particle hydrodynamics (SPH) method $[6,7]$, the reproducing kernel particle methods (RKPM) [8], the hpclouds method [9], the element-free Galerkin method (EFG) [10], the meshless local Petrov-Galerkin (MLPG) approach [11], the boundary node method (BNM) [12-14], the boundary element-free method (BEFM) [15], the hybrid boundary node method (hybrid BNM) [16-20], the Galerkin boundary node method (GBNM) [21], the boundary face method (BFM) $[22,23]$, and the boundary point interpolation method [24].

Meshless methods only require the scattered points in the domain or on the boundary, and the unknown fields are approximated by some approximation methods, of which the moving least-square (MLS) approximation [25] is the most commonly used. However, the MLS approximation does not have the delta function property and thus generates certain difficulties in many meshless methods, such as in the $\mathrm{BNM}$ and hybrid BNM, wherein the boundary conditions cannot be directly applied as in the BEM and requires the application of new sets of virtual nodal values to overcome this problem. Lancaster and Salkauskas further developed an interpolating moving least-square (IMLS) method, wherein specific singular functions were used as weight functions [25], thereby resulting in the computation of the inverse of the singular matrix and a reduction in the computational efficiency. Wang et al. [26, 27] recently proposed an improved interpolating moving least-square (IIMLS) method. It was applied in the improved interpolating element-free Galerkin method (IIEFG) [26] and the improved interpolating boundary element-free method (IIBEFM) [27]. Li [28] proposed the application of the IIMLS method into the boundary elementfree method (BEFM) for 3D potential problems and discussed the details of the refurbished IIMLS method. The IIMLS method has overcome drawbacks within the MLS and IMLS. Firstly, the shape functions obtained by the IIMLS method 
exhibit the delta function property, thereby resulting in easier boundary conditions implementation. Secondly, the weight function does not have any restrictions and any weight functions used in the MLS can be directly used in the IIMLS method. Therefore, the present study applied the IIMLS method as the approximation method to construct the shape functions of the proposed meshless method.

Most meshless methods, such as the EFG and BNM, cannot avoid the integral cells used in the approximation of the geometry. These cells are only applied for integration purposes and should be distinguished from the employed elements. However, models with complicated geometries often exhibit additional discretization errors because the integral cells are not always able to reproduce the exact geometry, which is a similar problem observed in the FEM or BEM with elements. Therefore, meshless methods with integral cells cannot overcome the second above-mentioned FEM- or BEM-related problem.

Hughes et al. [29] initially proposed an isogeometric analysis (IGA) that has recently received widespread research attention. The same parametric functions used in CAD to model the geometry are also used to approximate unknown fields in numerical analyses, of which most take the form of nonuniform rational B-splines (NURBS). In the IGA, the geometry of the model can be exactly preserved to avoid discretization errors. The idea has also been applied in other numerical methods, such as in the boundary element method $[2,30-34]$ and the boundary face method [35]. The present study focused on an improved interpolating boundary node method (IIBNM) based on the parameter space for $2 \mathrm{D}$ potential problems, also called the improved interpolating boundary element-free method (IIBEFM). Unlike the wellknown isogeometric analysis in FEM or BEM, the present study only applied the parametric function to reproduce the exact geometry and approximated the unknown fields by the IIMLS method. Thus, each curve only requires the parametric function, thereby characterizing the presented method as a general meshless method that is independent of the type of parametric function. Compared with IIBNM (IIBEFM), the proposed method used the parametric function to reproduce the exact geometry, and the integrations were performed in the parameter space. Thus, the discretization error was avoided. In addition, the IIMLS was also performed in parameter space and the curve lengths between boundary points were not needed.

This paper is organized as follows. The improved interpolating moving least-square method is first introduced in the second section, followed by the parameter spacederived cells in the third section. The fourth section presents the formulations of the IIBNM for 2D potential problems. Finally, some numerical examples are presented to show the accuracy of the proposed method.

\section{The Improved Interpolating Moving Least-Square Method}

Suppose $\mathbf{x}=\left\{x_{1}, \ldots, x_{d}\right\} \in \Omega$ is a point, $\Omega$ is a bounded domain in space $\mathbb{R}^{d}(d=1,2,3)$, and $\mathbf{y}=\left\{y_{1}, \ldots, y_{d}\right\}$ is a point in the local approximation domain $R(\mathbf{x})$ of $\mathbf{x}$, which can either be an evaluation point $\mathbf{x}$ or be a nodal point $\mathbf{y}_{i} \cdot n$ is the number of nodal points $\mathbf{y}_{i}$ in the domain of $R(\mathbf{x})$. For a given function $u(\mathbf{x})$, it can be approximated by IIMLS [26, 27] as

$$
u(\mathbf{x})=\psi(\mathbf{x}, \mathbf{x}) \mathbf{u}+\sum_{k=1}^{n} v\left(\mathbf{x}, \mathbf{y}_{k}\right) u\left(\mathbf{y}_{k}\right)=\boldsymbol{\Phi}(\mathbf{x}) \mathbf{u},
$$

where

$$
\begin{aligned}
\mathbf{u} & =\left[u\left(\mathbf{y}_{1}\right), u\left(\mathbf{y}_{2}\right), \ldots, u\left(\mathbf{y}_{n}\right)\right]^{T}, \\
\mathbf{\Phi}(\mathbf{x}) & =\psi(\mathbf{x}, \mathbf{x})+\mathbf{v}(\mathbf{x}), \\
\psi(\mathbf{x}, \mathbf{x}) & =\widehat{\mathbf{g}}(\mathbf{x}, \mathbf{x}) \mathbf{A}^{-1}(\mathbf{x}) \mathbf{B}(\mathbf{x}), \\
\mathbf{v}(\mathbf{x}) & =\left[v\left(\mathbf{x}, \mathbf{y}_{1}\right), v\left(\mathbf{x}, \mathbf{y}_{2}\right), \ldots, v\left(\mathbf{x}, \mathbf{y}_{n}\right)\right], \\
\widehat{\mathbf{g}}(\mathbf{x}, \mathbf{y}) & =\left[g_{2}(\mathbf{x}, \mathbf{y}), g_{3}(\mathbf{x}, \mathbf{y}), \ldots, g_{m}(\mathbf{x}, \mathbf{y})\right], \\
A_{l j}(\mathbf{x}) & =\sum_{i=1}^{n} w\left(\mathbf{x}-\mathbf{y}_{i}\right) g_{j}\left(\mathbf{x}, \mathbf{y}_{i}\right) g_{l}\left(\mathbf{x}, \mathbf{y}_{i}\right), \\
B_{l k}(\mathbf{x}) & =\sum_{i=1}^{n} w\left(\mathbf{x}-\mathbf{y}_{i}\right) g_{l}\left(\mathbf{x}, \mathbf{y}_{i}\right)\left[\delta_{i k}-v\left(\mathbf{x}, \mathbf{y}_{k}\right)\right], \\
g_{j}(\mathbf{x}, \mathbf{y}) & =p_{j}(\mathbf{y})-\sum_{i=1}^{n} v\left(\mathbf{x}, \mathbf{y}_{i}\right) p_{j}\left(\mathbf{y}_{i}\right), \\
\nu\left(\mathbf{x}, \mathbf{y}_{i}\right) & =\frac{\zeta\left(\mathbf{x}, \mathbf{y}_{i}\right)}{\sum_{k=1}^{n} \zeta\left(\mathbf{x}, \mathbf{y}_{k}\right)}, \\
\zeta\left(\mathbf{x}, \mathbf{y}_{k}\right) & =\frac{\prod_{i=1, i \neq k}^{n}\left\|\mathbf{x}-\mathbf{y}_{i}\right\|^{2}}{\prod_{i=1, i \neq k}^{n}\left\|\mathbf{y}_{k}-\mathbf{y}_{i}\right\|^{2}} .
\end{aligned}
$$

In (7) and (8), $w\left(\mathbf{x}-\mathbf{y}_{i}\right)$ are weight functions. $\left\{p_{j}(\mathbf{x})\right\}_{j=1}^{m}$ is a given set of basis functions with $p_{1}(\mathbf{x}) \equiv 1$.

In (11), for $\mathbf{x}=\mathbf{y}_{l}$, one can have

$$
\zeta\left(\mathbf{y}_{l}, \mathbf{y}_{k}\right)=\frac{\prod_{i=1, i \neq k}^{n}\left\|\mathbf{y}_{l}-\mathbf{y}_{i}\right\|^{2}}{\prod_{i=1, i \neq k}^{n}\left\|\mathbf{y}_{k}-\mathbf{y}_{i}\right\|^{2}}
$$

If $l=k, \zeta\left(\mathbf{y}_{l}, \mathbf{y}_{k}\right)=1$. If $l \neq k$, there was a node $\mathbf{y}_{l}=\mathbf{y}_{i}$, and thus $\zeta\left(\mathbf{y}_{l}, \mathbf{y}_{k}\right)=0$. Then one can have $\sum_{k=1}^{n} \zeta\left(\mathbf{y}_{l}, \mathbf{y}_{k}\right)=1$. Finally, from (10) and (11), one can note that

$$
\begin{gathered}
v\left(\mathbf{y}_{i}, \mathbf{y}_{j}\right)=\delta_{i j}= \begin{cases}1, & i=j \\
0, & i \neq j,\end{cases} \\
\sum_{i=1}^{n} v\left(\mathbf{x}, \mathbf{y}_{i}\right)=1, \quad \mathbf{x} \in \Omega .
\end{gathered}
$$

The shape function of the IIMLS has the following properties [28].

Property 1 (interpolating property).

$$
\Phi_{i}\left(\mathbf{x}_{j}\right)=\delta_{i j}= \begin{cases}1, & i=j \\ 0, & i \neq j .\end{cases}
$$


Property 2 (reproducing property).

$$
\sum_{i=1}^{n} \Phi_{i}(\mathbf{x}) p_{j}\left(\mathbf{y}_{i}\right)=p_{j}(\mathbf{x}),
$$

where $j$ is defined from 1 to $m$.

\section{Improved Interpolating Boundary Node Method for 2D Potential Problems}

The well-known regularized boundary integral equation for 2D potential problems can be written as follows:

$$
\begin{aligned}
\mathbf{0}= & \int_{\Gamma} u^{*}(\mathbf{x}, \mathbf{y}) q(\mathbf{y}) \mathrm{d} \Gamma(\mathbf{y}) \\
& -\int_{\Gamma} q^{*}(\mathbf{x}, \mathbf{y})[u(\mathbf{y})-u(\mathbf{x})] \mathrm{d} \Gamma(\mathbf{y}),
\end{aligned}
$$

where $\Gamma$ is the boundary of the bound domain $\Omega, \mathbf{x}$ and $\mathbf{y}$ are points on the boundary, and $u$ and $q$ are the potential and normal flux on the boundary, respectively. $u^{*}(\mathbf{x}, \mathbf{y})$ and $q^{*}(\mathbf{x}, \mathbf{y})$ are fundamental solutions of the Laplace equation, which can be written in the $2 \mathrm{D}$ case as follows:

$$
\begin{aligned}
u^{*}(\mathbf{x}, \mathbf{y}) & =\frac{1}{2 \pi} \ln \left(\frac{1}{r(\mathbf{x}, \mathbf{y})}\right), \\
q^{*}(\mathbf{x}, \mathbf{y}) & =\frac{\partial u^{*}(\mathbf{x}, \mathbf{y})}{\partial \mathbf{n}(\mathbf{y})},
\end{aligned}
$$

where $r(\mathbf{x}, \mathbf{y})=\|\mathbf{x}-\mathbf{y}\|$ is the distance between $\mathbf{x}$ and $\mathbf{y}$ and $\mathbf{n}$ is the unit outward normal at the boundary point.

The field of $u$ and $q$ at the boundary can be separately interpolated by the IIMLS method within the parameter space of each curve. Namely, the influence domain of an evaluation point is truncated at the corner of the boundary. Supposing that a boundary has $n$ distributed nodes $\mathbf{x}_{i}\left(\xi_{i}\right)$, then the field of $u$ and $q$ on the boundary can be interpolated by the IIMLS method in the parameter space as follows:

$$
\begin{aligned}
& u(\mathbf{x})=u(\mathbf{x}(\xi))=\sum_{i=1}^{n} \Phi_{i}(\xi) u_{i}=\boldsymbol{\Phi} \mathbf{u} \\
& q(\mathbf{x})=q(\mathbf{x}(\xi))=\sum_{i=1}^{n} \Phi_{i}(\xi) q_{i}=\boldsymbol{\Phi} \mathbf{q}
\end{aligned}
$$

where $\Phi_{i}(\xi)$ is the contribution from node $\xi_{i}$ to the evaluated point $\xi$ obtained in the $1 \mathrm{D}$ parameter space. $\Phi_{i}(\xi)=0$ if $\xi_{i}$ is out of the influence domain of $\xi . u_{i}$ and $q_{i}$ are nodal values of $u$ and $q$ at $\xi_{i}$. In the real computation, the parameter coordinate $\xi$ is defined on each smooth curve separately, and (18) are performed only on the local nodes in the influence domain of $\xi$. In parameter space, $\Phi$ can be written as

$$
\Phi=\Phi(\xi)=\psi(\xi, \xi)+\mathbf{v}(\xi) .
$$

Substituting (18) into (16) presents

$$
\begin{aligned}
\int_{\Gamma} u^{*}(\mathbf{x}(\varsigma), \mathbf{y}(\xi)) \sum_{i=1}^{n} \Phi_{i}(\xi) q_{i} \mathrm{~d} \Gamma(\mathbf{y}) \\
=\int_{\Gamma} q^{*}(\mathbf{x}(\varsigma), \mathbf{y}(\xi)) \\
.\left[\sum_{i=1}^{n} \Phi_{i}(\xi) u_{i}-\sum_{i=1}^{n} \Phi_{i}(\varsigma) u_{i}\right] \mathrm{d} \Gamma(\mathbf{y}),
\end{aligned}
$$

where $\varsigma$ and $\xi$ are the parameter coordinates of points $\mathbf{x}$ and $\mathrm{y}$, respectively.

By discretizing the boundary into cells in the parameter space, (20) can be rewritten as follows:

$$
\begin{gathered}
\sum_{k=1}^{N} \int_{\Gamma_{k}} u^{*}(\mathbf{x}(\varsigma), \mathbf{y}(\xi)) \sum_{i=1}^{n} \Phi_{i}(\xi) q_{i} J \mathrm{~d} \xi \\
=\sum_{k=1}^{N} \int_{\Gamma_{k}} q^{*}(\mathbf{x}(\varsigma), \mathbf{y}(\xi)) \\
\cdot\left[\sum_{i=1}^{n} \Phi_{i}(\xi) u_{i}-\sum_{i=1}^{n} \Phi_{i}(\varsigma) u_{i}\right] J \mathrm{~d} \xi,
\end{gathered}
$$

where $\Gamma_{k}$ is the $k$ th cell and $J$ is defined as $J=$ $\sqrt{\left(\partial x_{1} / \partial \xi\right)^{2}+\left(\partial x_{2} / \partial \xi\right)^{2}}$.

For each node $\mathbf{x}_{j}\left(\xi_{j}\right)$ on the boundary, an equation like (21) can be presented as follows:

$$
\begin{gathered}
\sum_{k=1}^{N} \int_{\Gamma_{k}} u^{*}\left(\mathbf{x}_{j}\left(\xi_{j}\right), \mathbf{y}(\xi)\right) \sum_{i=1}^{n} \Phi_{i}(\xi) q_{i} J \mathrm{~d} \xi \\
=\sum_{k=1}^{N} \int_{\Gamma_{k}} q^{*}\left(\mathbf{x}_{j}\left(\xi_{j}\right), \mathbf{y}(\xi)\right) \\
\cdot\left[\sum_{i=1}^{n} \Phi_{i}(\xi) u_{i}-\sum_{i=1}^{n} \Phi_{i}\left(\xi_{j}\right) u_{i}\right] J \mathrm{~d} \xi .
\end{gathered}
$$

Since $\Phi_{i}\left(\xi_{j}\right)$ exhibits the interpolating property, (22) can be rewritten as follows:

$$
\begin{gathered}
\sum_{k=1}^{N} \int_{\Gamma_{k}} u^{*}\left(\mathbf{x}_{j}\left(\xi_{j}\right), \mathbf{y}(\xi)\right) \sum_{i=1}^{n} \Phi_{i}(\xi) q_{i} J \mathrm{~d} \xi \\
=\sum_{k=1}^{N} \int_{\Gamma_{k}} q^{*}\left(\mathbf{x}_{j}\left(\xi_{j}\right), \mathbf{y}(\xi)\right) \sum_{i=1}^{n} \Phi_{i}(\xi) u_{i} J \mathrm{~d} \xi \\
\quad-\sum_{k=1}^{N} \int_{\Gamma_{k}} q^{*}\left(\mathbf{x}_{j}\left\{\xi_{j}\right\}, \mathbf{y}\{\xi\}\right) u_{j} J \mathrm{~d} \xi .
\end{gathered}
$$

Finally, (21) can be written in matrix form as follows:

$$
\mathbf{H u}=\mathbf{G q},
$$




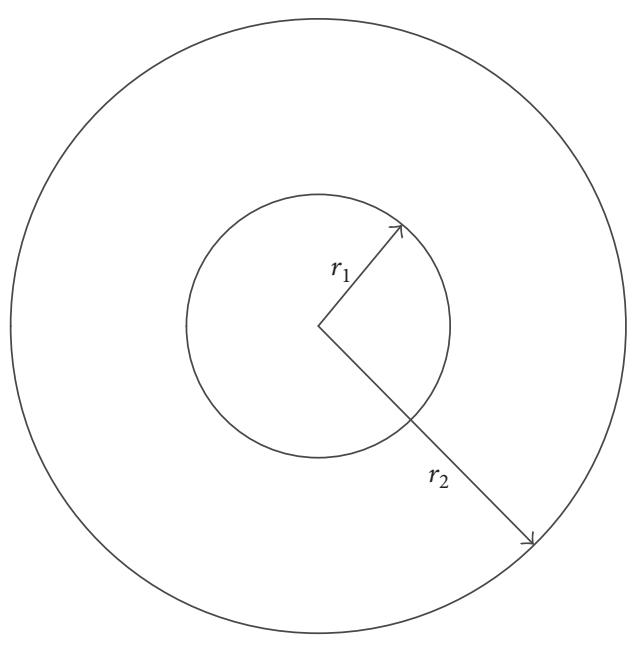

(a)

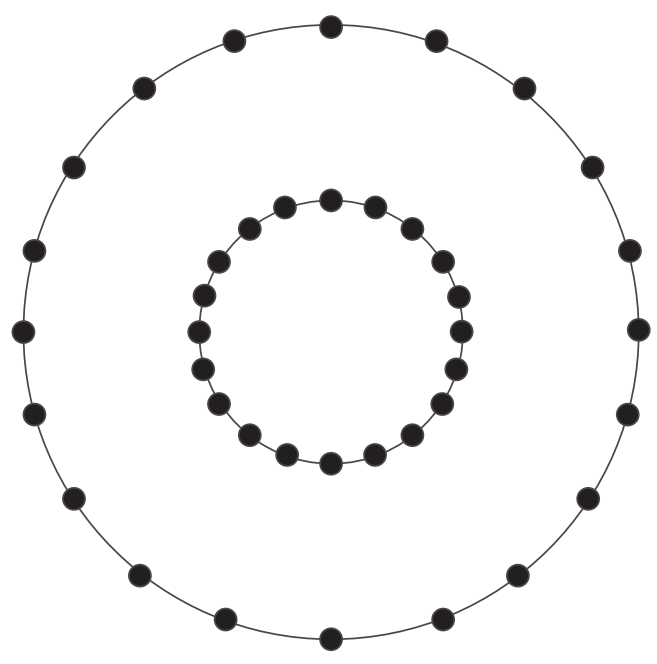

(b)

Figure 1: A concentric circular ring.

where

$$
\begin{aligned}
H_{j i}= & \sum_{\Gamma_{k} \in K_{i}} \int_{\Gamma_{k}} q^{*}\left(\mathbf{x}_{j}\left(\xi_{j}\right), \mathbf{y}(\xi)\right) \Phi_{i}(\xi) J \mathrm{~d} \xi \\
& -\delta_{j i} \sum_{\Gamma_{k} \in K_{j}} \int_{\Gamma_{k}} q^{*}\left(\mathbf{x}_{j}\left(\xi_{j}\right), \mathbf{y}(\xi)\right) J \mathrm{~d} \xi, \\
G_{j i}= & \sum_{\Gamma_{k} \in K_{i}} \int_{\Gamma_{k}} u^{*}\left(\mathbf{x}_{j}\left(\xi_{j}\right), \mathbf{y}(\xi)\right) \Phi_{i}(\xi) J \mathrm{~d} \xi, \\
\mathbf{u}= & {\left[u_{1}, u_{2}, \ldots, u_{n}\right]^{T}, } \\
\mathbf{q}= & {\left[q_{1}, q_{2}, \ldots, q_{n}\right]^{T} . }
\end{aligned}
$$

In (25) and (26), $K_{i}$ is the set of cells that contributes to $\Phi_{i}(\xi)$ and $K_{j}$ is the set of cells that contain node $\mathbf{x}_{j}\left(\xi_{j}\right)$. Equations (24) to (28) are very similar to the equations in the BEM. Actually, the methods used in the BEM for the treatment of singular integrals can also be applied in the IIBNM.

The present study employed the following basis functions $\left\{p_{j}(\xi)\right\}_{j=1}^{m}$ :

$$
\mathbf{p}^{T}=\left[1, \xi, \xi^{2}\right]^{T}, \quad m=3
$$

In addition, the Gaussian weight function was applied as the weight function in the IIMLS method.

\section{Numerical Examples}

Numerical examples were examined to justify the accuracy of the proposed method. For the purpose of error estimation, a formula is defined as follows:

$$
e=\frac{1}{|w|_{\max }} \sqrt{\frac{1}{N} \sum_{i=1}^{N}\left(w_{i}^{(e)}-w_{i}^{(n)}\right)^{2}},
$$

where $w_{i}^{(e)}$ and $w_{i}^{(n)}$ refer to the exact and numerical solutions, respectively, and $|w|_{\max }$ is the maximum value of $w$ over $N$ nodes. In this paper, $w$ are the potential $u$ or the derivatives of $u$.

Example 1 (Dirichlet problems on a circular ring). This example presents concentric a circular ring centered at $x_{1}=0$ and $x_{2}=0$ with radii of $r_{1}=1.0$ and $r_{2}=2.0$, which is shown in Figure 1(a). Dirichlet boundary conditions were imposed on the boundaries and the following solutions were employed as the analytical solutions.

(i) Linear solution is

$$
u=x_{1}+x_{2} \text {. }
$$

(ii) Quadratic solution-1 is

$$
u=x_{1} x_{2}
$$

(iii) Quadratic solution-2 is

$$
u=x_{1}^{2}-x_{2}^{2}
$$

(iv) Cubic solution is

$$
u=x_{1}^{3}+x_{2}^{3}-3 x_{1}^{2} x_{2}-3 x_{1} x_{2}^{2} .
$$

Five sets of nodes were employed to discretize the model: (a) 40 nodes (Figure 1(b)), (b) 80 nodes, (c) 160 nodes, (d) 320 nodes, and (e) 640 nodes. The relative errors of $u, \partial u / \partial x_{1}$, and $\partial u / \partial x_{2}$ with respect to the number of nodes are plotted in Figures 2, 3, and 4, respectively. The proposed method exhibited good convergence rates for all the four different analytical solutions and presented very high accuracy.

Example 2 (Dirichlet problems on an elliptical ring). The present example examined an elliptical ring with Dirichlet boundary conditions, as presented in Figure 5(a). The inner 


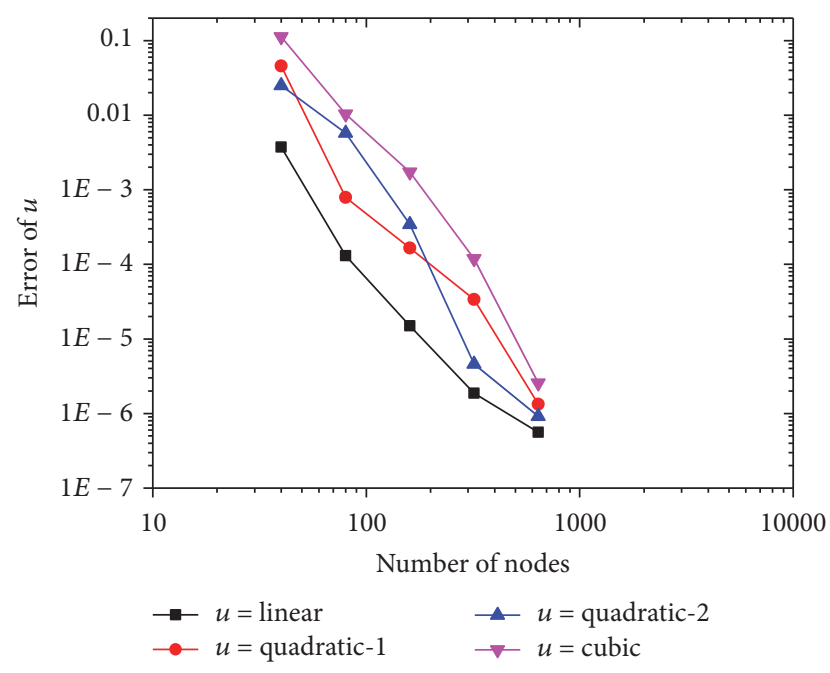

FIgURE 2: Relative error of $u$ for Example 1.

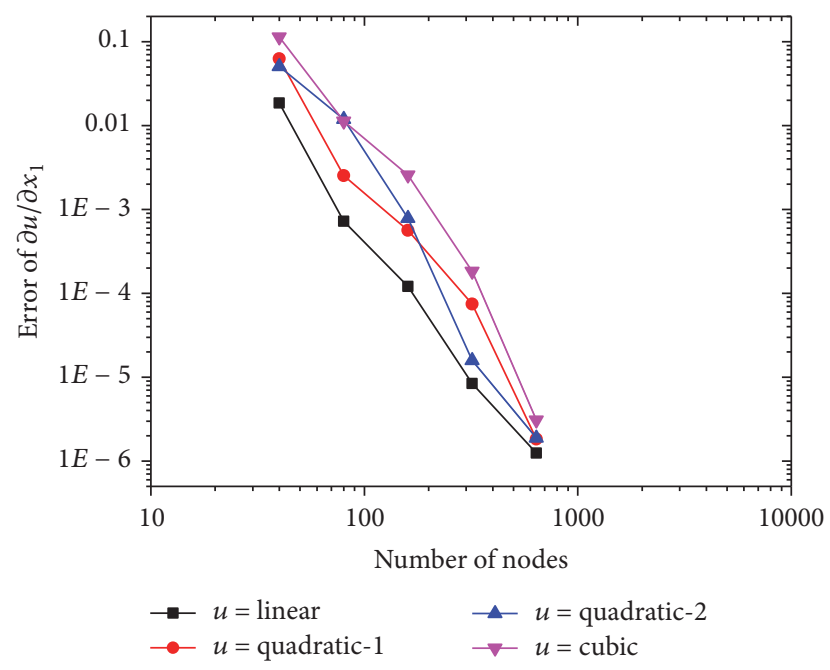

FIgURE 3: Relative error of $\partial u / \partial x_{1}$ for Example 1.

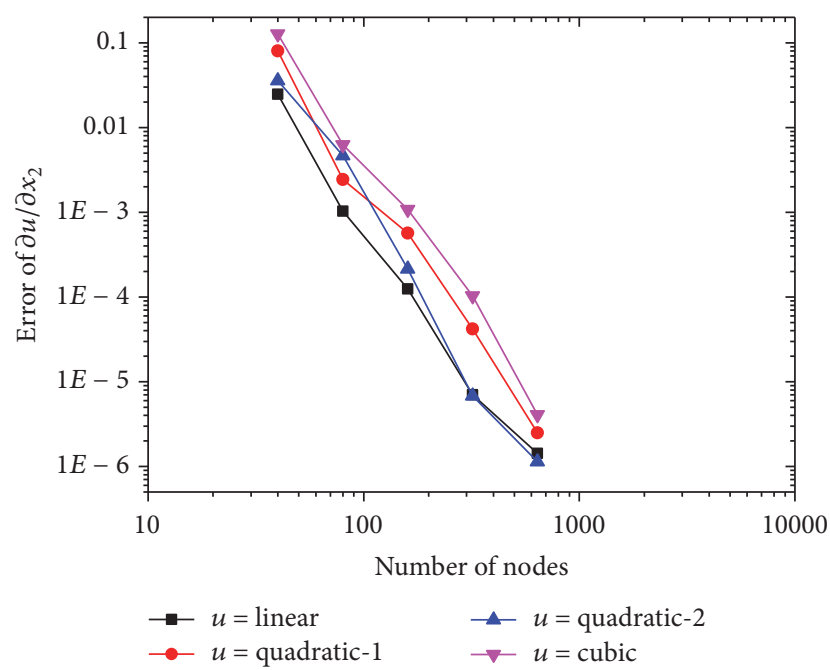

FIgURE 4: Relative error of $\partial u / \partial x_{2}$ for Example 1. ellipse had a major radius and minor radius of 2 and 1 , whereas the outer ellipse had a major radius and minor radius of 8 and 4 . follows:

The analytical solution for this example was derived as

$$
u=\sinh x_{1} \sin x_{2} .
$$

Two discretization methods were applied to test the proposed method. In discretization method 1 (Figure 5(b)), the numbers of nodes distributed on the inner and outer curves were the same and seven sets of nodes were used to discretize the model presented in Table 1. Conversely, discretization method 2 (Figure 5(c)) exhibited nearly equal curve distances between the two nearest nodes, of which the number of nodes for each discretization is presented in Table 1 .

The relative errors of $u, \partial u / \partial x_{1}$, and $\partial u / \partial x_{2}$ with respect to the number of nodes for the two different discretization methods are plotted in Figures 6 and 7, wherein the error exhibited a decrease following an increase in the number of nodes. Both discretization methods exhibited a high convergence rate.

The contour of potential $u$ computed by total 1940 nodes was shown in Figure 8, and the results computed by traditional BEM with 1938 linear elements were also plotted for the purpose of comparison. It could be observed that the results of the proposed methods have good agreement with those obtained by BEM.

Example 3 (potential flow problem). The present example considered the potential flow problem around a cylinder of radius $a=1$ in an infinite domain. Here, due to the symmetry of the problem, only part of the upper left quadrant of the field was modeled as presented in Figure 9, where $L=4$ and $W=2$ in the figure. The exact solution for this problem is given as follows [36]:

$$
u=x_{2}\left[1-\frac{a^{2}}{x_{2}^{2}+\left(x_{1}-L\right)^{2}}\right],
$$

where $u$ represents the stream function.

The boundary conditions of this problem are as follows:

$$
\begin{aligned}
& \text { Curve (1): } u=x_{2}\left(x_{2}^{2}+15\right) / x_{2}^{2}+16 . \\
& \text { Curve (2): } u=0 . \\
& \text { Curve (3): } u=0 . \\
& \text { Curve (4): } \partial u / \partial n=0 . \\
& \text { Curve (5): } u=\left[6+2\left(x_{1}-4\right)^{2}\right] /\left[4+\left(x_{1}-4\right)^{2}\right] .
\end{aligned}
$$

The relative errors of $u, q_{1}=\partial u / \partial x_{1}$, and $q_{2}=$ $\partial u / \partial x_{2}$ were computed from 115 nodes in the domain, as plotted in Figure 10. According to Figure 10, the proposed method exhibited a high accuracy to obtain a good rate of convergence.

The contours of stream function $u$ computed by 405 boundary nodes are shown in Figure 11. BEM with 400 linear elements is also applied to solve this problem. Good agreement can be observed in these two methods. 
TABLE 1: Information of the discretization.

\begin{tabular}{lcccccccc}
\hline & Nodes on inner curve & 31 & 61 & 122 & 244 & 486 & 970 & 1939 \\
Discretization method 1 & Nodes on outer curve & 31 & 61 & 122 & 244 & 486 & 970 & 1939 \\
& Total nodes & 62 & 122 & 244 & 486 & 972 & 1940 & 3878 \\
\hline & Nodes on inner curve & 13 & 25 & 49 & 98 & 195 & 389 & 776 \\
Discretization method 2 & Nodes on outer curve & 49 & 98 & 195 & 399 & 776 & 1551 & 3101 \\
& Total nodes & 62 & 123 & 244 & 487 & 971 & 1940 & 3877 \\
\hline
\end{tabular}

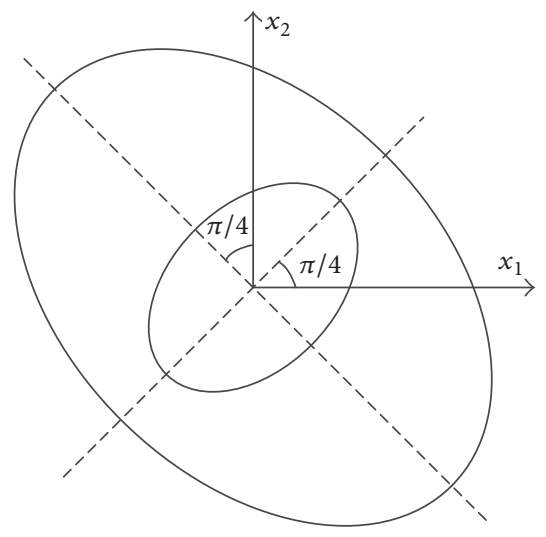

(a)

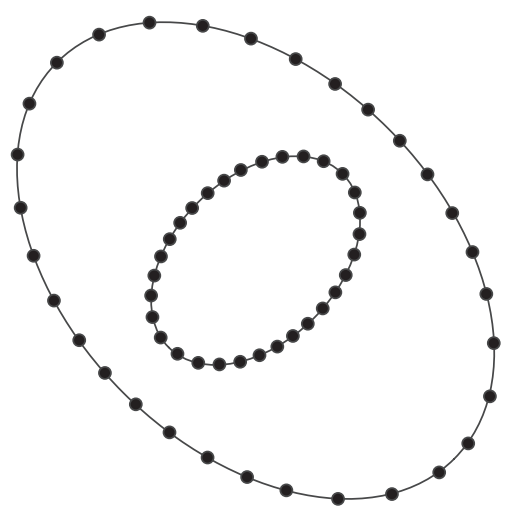

(b)

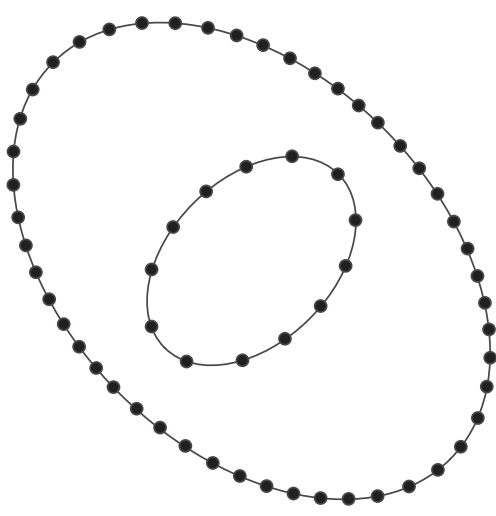

(c)

Figure 5: An elliptical ring.

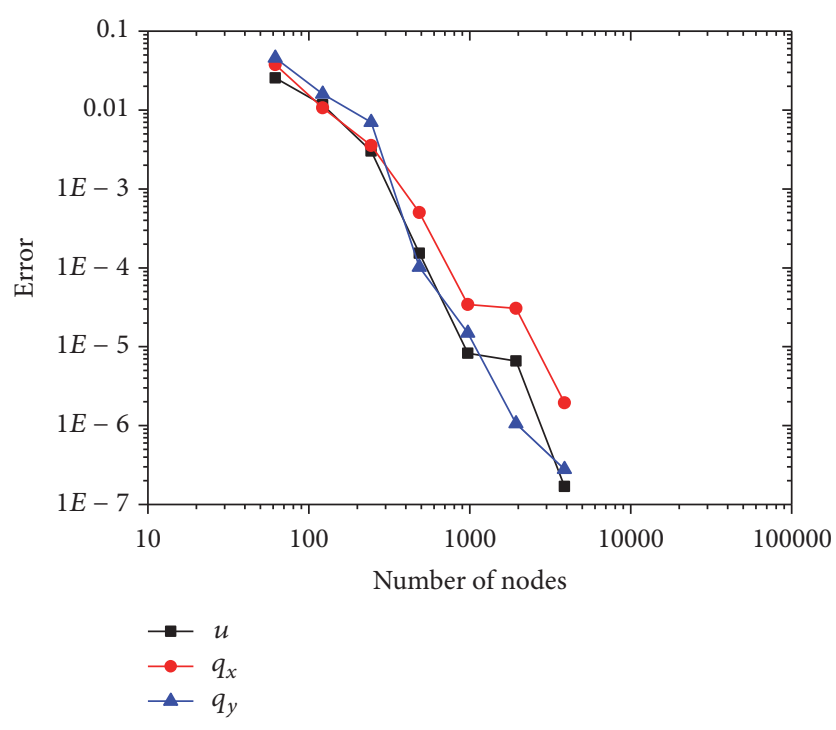

FIgURE 6: Relative error for discretization method 1 in Example 2.

Example 4 (mixed problem on a complex model). The present example offers a complex model with mixed boundary conditions, as presented in Figure 12. Neumann boundary conditions were imposed on line $x_{2}= \pm 8$ and Dirichlet boundary conditions were imposed on the rest of the boundaries. The model employed the following analytical solution:

$$
u=e^{x_{1}} \sin x_{2}+e^{x_{2}} \sin x_{1}
$$

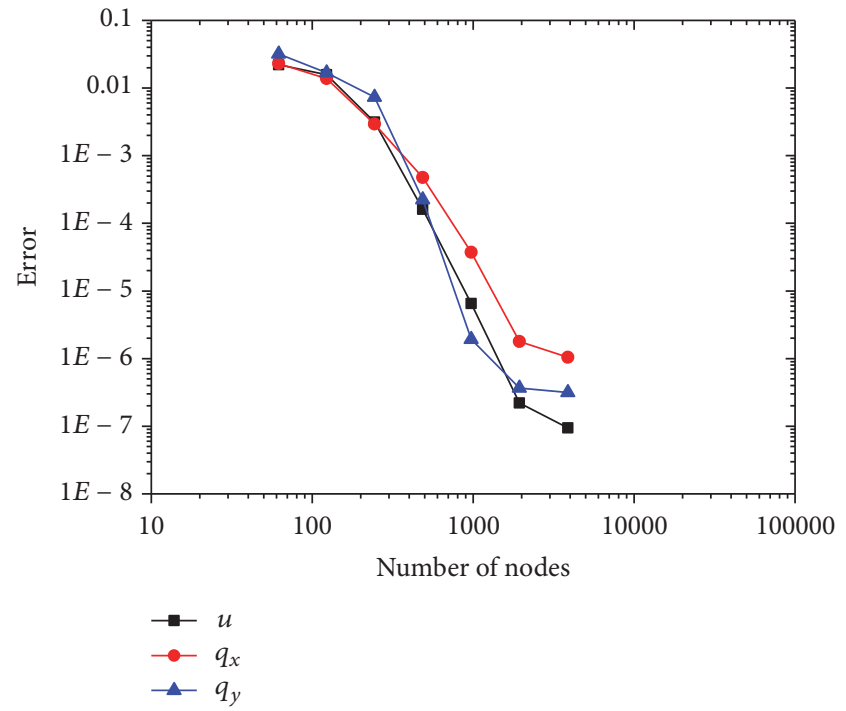

FIGURE 7: Relative error for discretization method 2 in Example 2.

A total of 737 nodes were distributed on the boundaries for the IIBNM. The BEM with a total of 714 linear elements was also applied to solve the problem. The numerical results of $u, \partial u / \partial x_{1}$, and $\partial u / \partial x_{2}$ on line $x_{2}=-1.0$ between points $\left\{x_{1}=1.0, x_{2}=-1.0\right\}$ and $\left\{x_{1}=8.5, x_{2}=-1.0\right\}$ are plotted in Figures 13, 14, and 15, respectively. The contour of $u$ was shown in Figure 16 and the results were compared with BEM. The figures exhibited very high accuracy of IIBNM. Likewise, the IIBNM results were well in agreement with those obtained by the BEM and the analytical solution. 


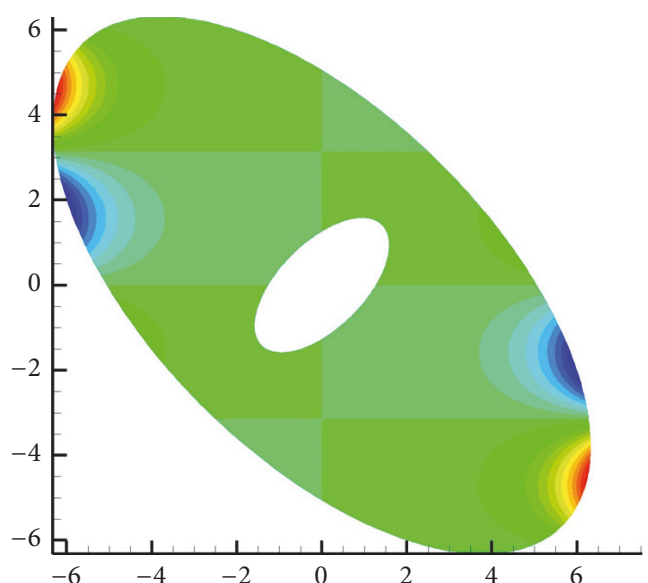

(a)

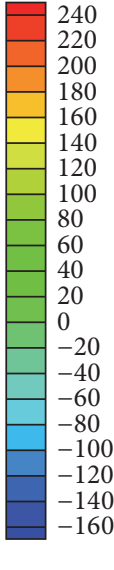

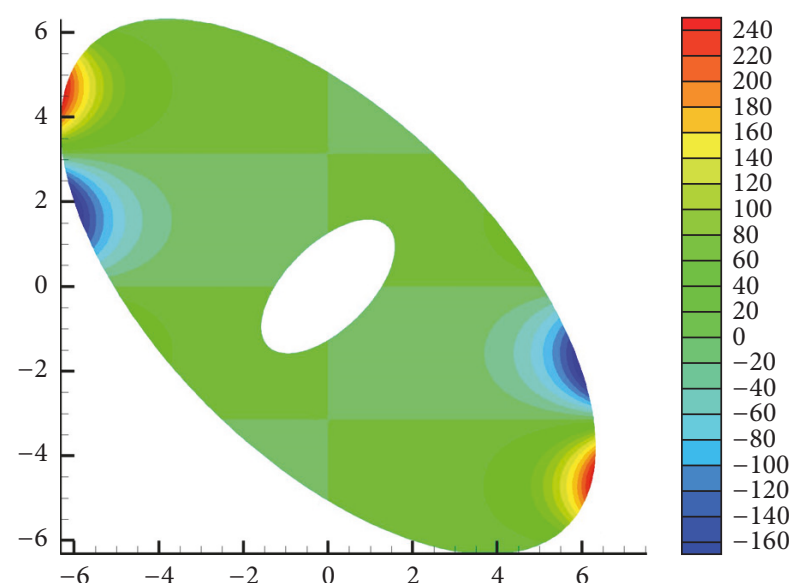

(b)

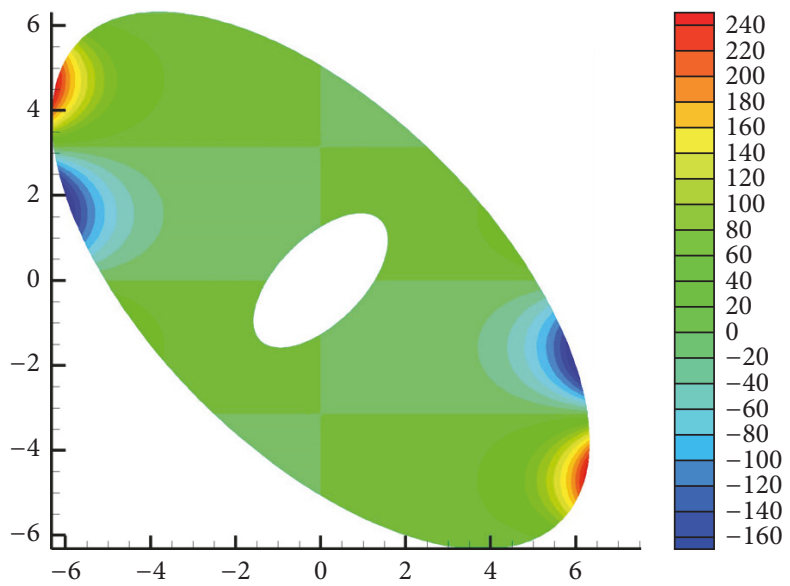

(c)

FIGURE 8: Contours of potential $u$ of the elliptical ring: (a) proposed method (discretization method 1); (b) proposed method (discretization method 2); (c) BEM.

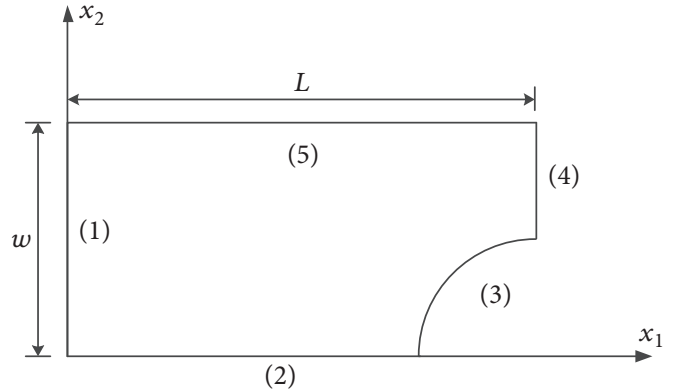

Figure 9: Model of flow around a cylinder.

\section{Conclusions}

The present study proposed a meshless improved interpolating boundary node method based on the parameter space. The improved interpolating moving least-square method was employed as the approximation method to construct the shape function of the meshless method. The shape functions exhibited delta function properties as compared to those obtained by the moving least-square method. In addition, any

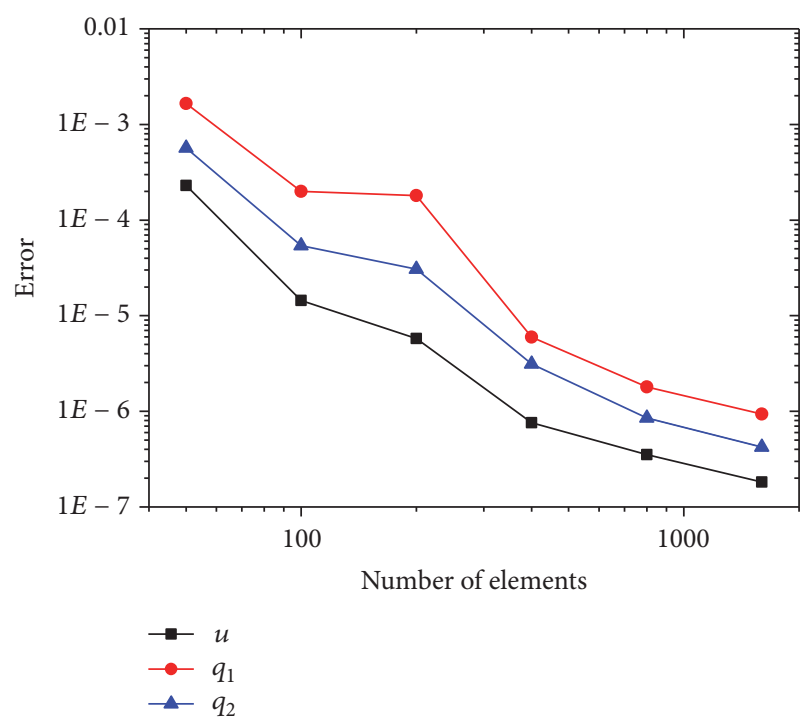

FIGURE 10: Relative error of for potential flow problem.

weight function used in the MLS was also applicable in the IIMLS method. 


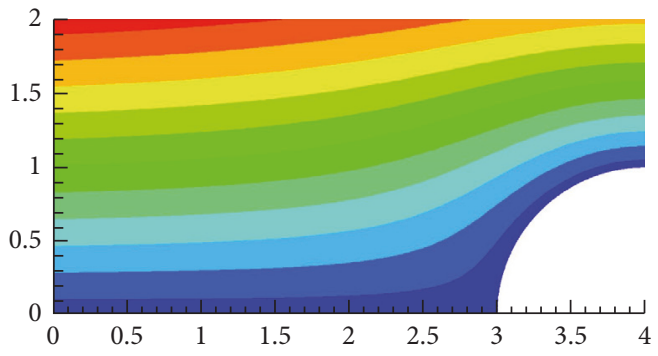

(a)

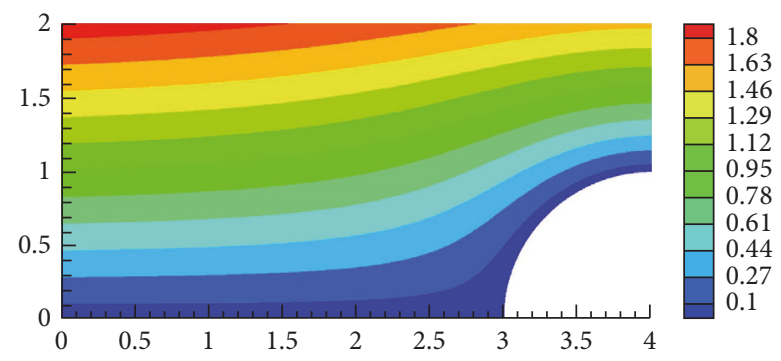

(b)

FIGURE 11: Contour of $u$ for potential flow problem: (a) proposed method and (b) BEM.

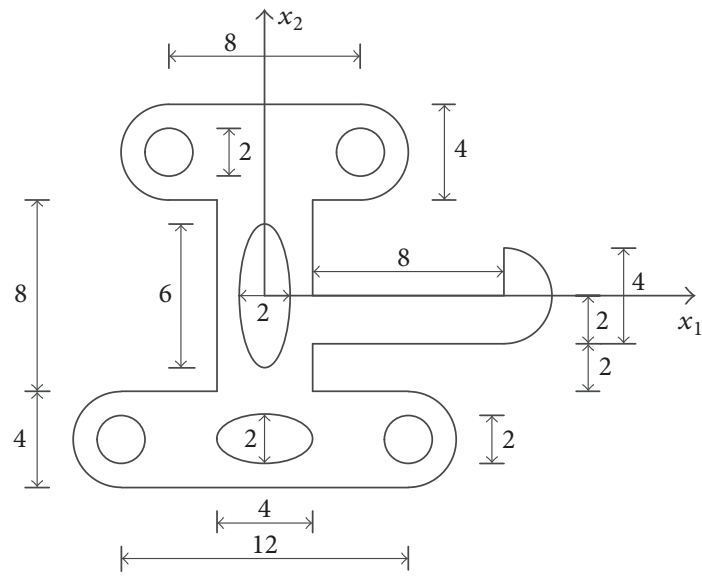

Figure 12: A complex model.

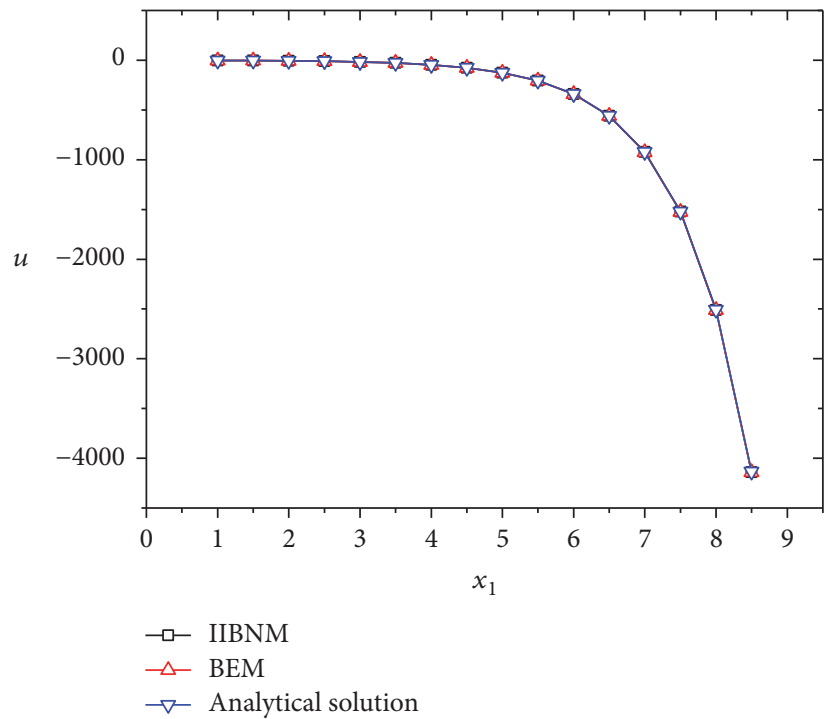

FIGURE 13: Results of $u$.

Cells were created in the parameter space, which are called parametric cells, to avoid the generation of integral cell discretization errors. The cells can produce exact geometry no matter the number of distributed nodes on the boundary. In addition, the IIMLS method was also performed in the

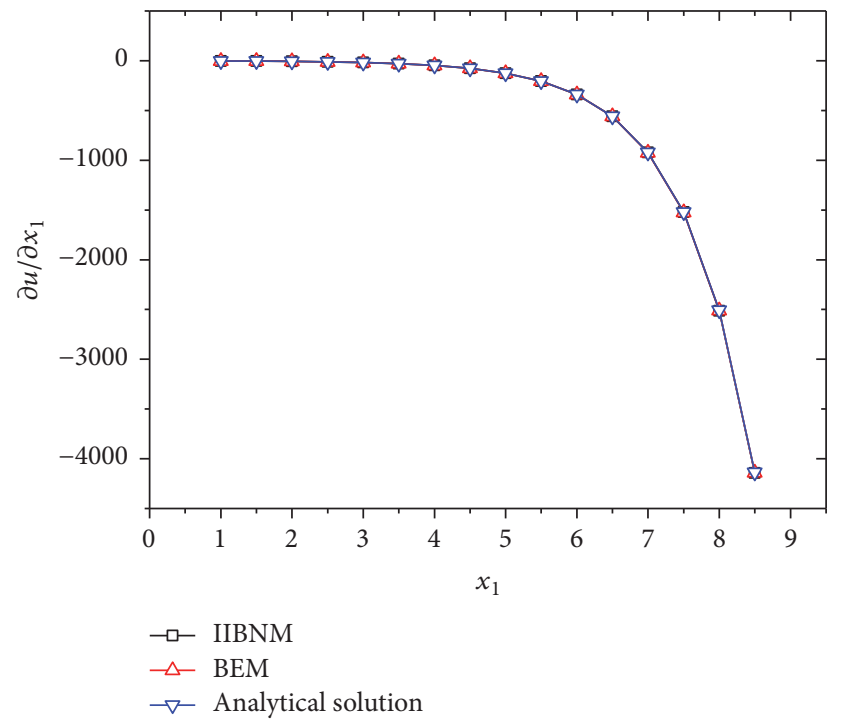

FIGURE 14: Results of $\partial u / \partial x_{1}$.

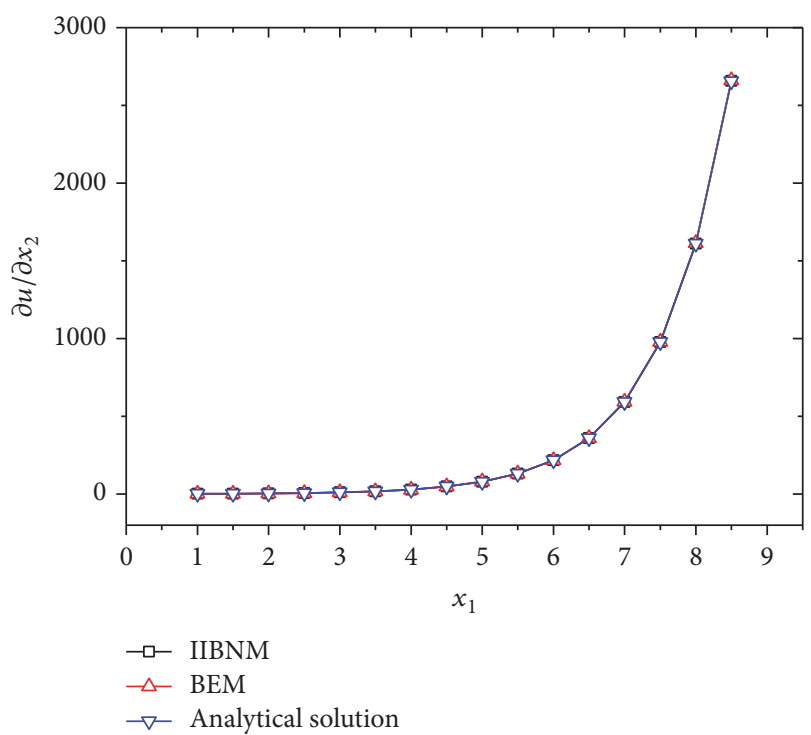

FIGURE 15: Results of $\partial u / \partial x_{2}$.

parameter space, thereby eliminating the evaluation for the curve length, which simplifies the procedures. 


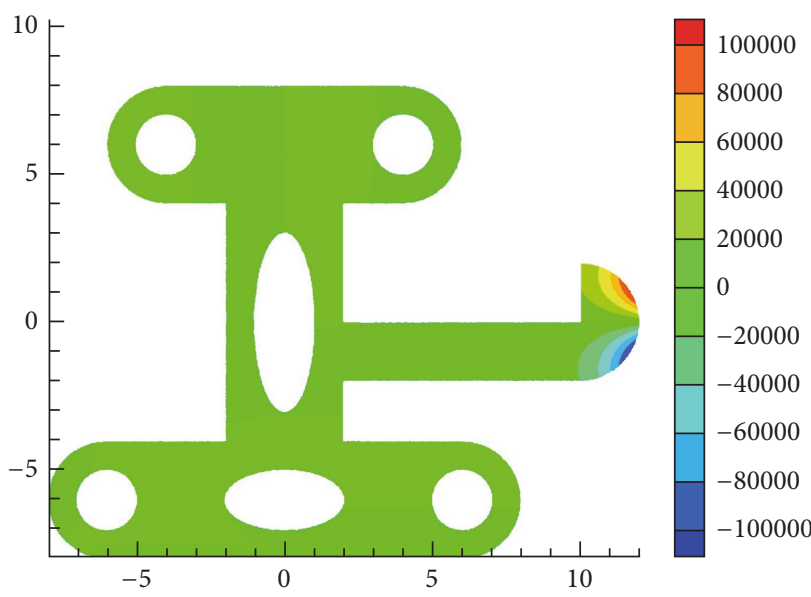

(a)

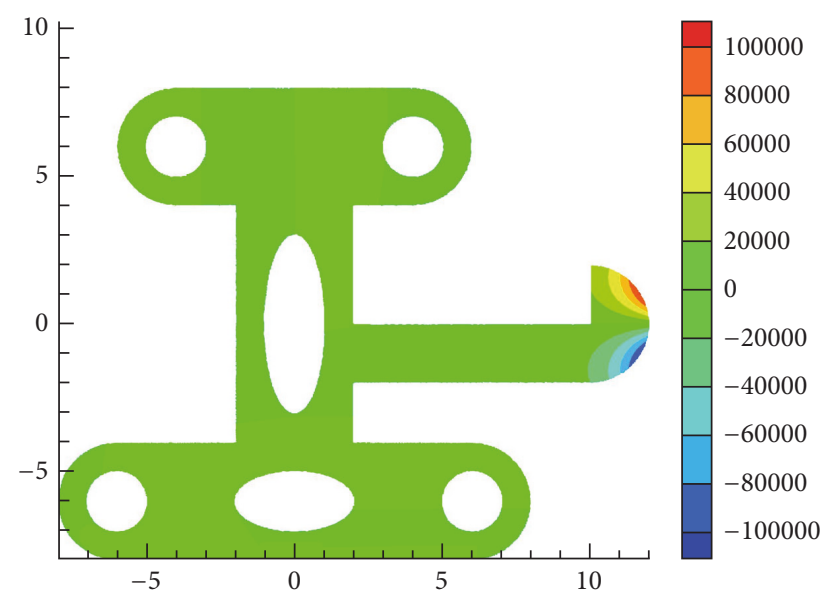

(b)

FIgURE 16: Contour of $u$ for Example 3: (a) proposed method and (b) BEM.

The proposed method can be extended to other problems and $3 \mathrm{D}$ problems. Coupling the presented method with the fast multipole method [37-39] for large-scale computations is under research. It is also possible to solve problems with body force by coupling the presented method with methods for domain integrals [40-42] in the BEM.

\section{Conflicts of Interest}

The authors declare that they have no conflicts of interest.

\section{Acknowledgments}

The research for this paper was supported by (1) the National Natural Science Foundation of China (Grants nos. 51708429, 51378404, and 51408444) and (2) Wuhan Institute of Technology Science Fund (Grant no. K201734).

\section{References}

[1] Z. Yao and H. Wang, "Some benchmark problems and basic ideas on the accuracy of boundary element analysis," Engineering Analysis with Boundary Elements, vol. 37, no. 12, pp. 16741692, 2013.

[2] F. Tan and Y.-Y. Jiao, "The combination of the boundary element method and the numerical manifold method for potential problems," Engineering Analysis with Boundary Elements, vol. 74, pp. 19-23, 2017.

[3] Q. Wang, W. Zhou, Y. Cheng, G. Ma, and X. Chang, "A line integration method for the treatment of $3 \mathrm{D}$ domain integrals and accelerated by the fast multipole method in the BEM," Computational Mechanics, vol. 59, no. 4, pp. 611-624, 2017.

[4] Q. Wang, W. Zhou, Y. Cheng, G. Ma, X. Chang, and Q. Huang, "The boundary element method with a fast multipole accelerated integration technique for 3D elastostatic problems with arbitrary body forces," Journal of Scientific Computing, vol. 71, no. 3, pp. 1238-1264, 2017.

[5] J.-H. Lv, X.-T. Feng, S.-J. Li, Q. Jiang, and H.-S. Guo, "Solid analysis of micron-sized thin structures with BEM for steady-state heat conduction," Engineering Analysis with Boundary Elements, vol. 71, pp. 11-19, 2016.
[6] L. B. Lucy, "A numerical approach to the testing of the fission hypothesis," The Astronomical Journal, vol. 82, pp. 1013-1024, 1977.

[7] R. A. Gingold and J. J. Monaghan, "Smoothed particle hydrodynamics-theory and application to non-spherical stars," Monthly Notices of The Royal Astronomical Society, vol. 181, pp. 375-389, 1977.

[8] W. K. Liu, S. Jun, and Y. F. Zhang, "Reproducing kernel particle methods," International Journal for Numerical Methods in Fluids, vol. 20, no. 8-9, pp. 1081-1106, 1995.

[9] T. J. Liszka, C. A. M. Duarte, and W. W. Tworzydlo, "hpMeshless cloud method," Computer Methods Applied Mechanics and Engineering, vol. 139, no. 1-4, pp. 263-288, 1996.

[10] T. Belytschko, Y. Y. Lu, and L. Gu, "Element-free Galerkin methods," International Journal for Numerical Methods in Engineering, vol. 37, no. 2, pp. 229-256, 1994.

[11] S. N. Atluri and T. Zhu, "A new meshless local Petrov-Galerkin (MLPG) approach in computational mechanics," Computational Mechanics, vol. 22, no. 2, pp. 117-127, 1998.

[12] Y. Xie Mukherjee and S. Mukherjee, "The boundary node method for potential problems," International Journal for Numerical Methods in Engineering, vol. 40, no. 5, pp. 797-815, 1997.

[13] J. H. Lv, Y. Miao, and H. P. Zhu, "Boundary node method based on parametric space for 2D elasticity," Engineering Analysis with Boundary Elements, vol. 37, no. 4, pp. 659-665, 2013.

[14] M. K. Chati and S. Mukherjee, "The boundary node method for three-dimensional problems in potential theory," International Journal for Numerical Methods in Engineering, vol. 47, no. 9, pp. 1523-1547, 2000.

[15] K. M. Liew, Y. Cheng, and S. Kitipornchai, "Boundary elementfree method (BEFM) and its application to two-dimensional elasticity problems," International Journal for Numerical Methods in Engineering, vol. 65, no. 8, pp. 1310-1332, 2006.

[16] Y. Miao, Q. Wang, H. Zhu, and Y. Li, “Thermal analysis of 3D composites by a new fast multipole hybrid boundary node method," Computational Mechanics, vol. 53, no. 1, pp. 77-90, 2014.

[17] Y. Miao, Z. Chen, Q. Wang, and H. Zhu, "Mechanical analysis of 3D composite materials by hybrid boundary node method," 
Computers, Materials and Continua, vol. 43, no. 1, pp. 49-73, 2014.

[18] Q. Wang, Y. Miao, and H. Zhu, "A new formulation for thermal analysis of composites by hybrid boundary node method," International Journal of Heat and Mass Transfer, vol. 64, pp. 322330, 2013.

[19] Q. Wang, Y. Miao, and H. Zhu, "A fast multipole hybrid boundary node method for composite materials," Computational Mechanics, vol. 51, no. 6, pp. 885-897, 2013.

[20] Q. Wang, Y. Miao, and H. Zhu, "Numerical simulation of heat conduction problems by a new fast multipole hybrid boundarynode method," Numerical Heat Transfer, Part B: Fundamentals, vol. 64, no. 6, pp. 436-459, 2013.

[21] X. Li and J. Zhu, "A Galerkin boundary node method and its convergence analysis," Journal of Computational and Applied Mathematics, vol. 230, no. 1, pp. 314-328, 2009.

[22] J. Zhang, X. Qin, X. Han, and G. Li, "A boundary face method for potential problems in three dimensions," International Journal for Numerical Methods in Engineering, vol. 80, no. 3, pp. 320-337, 2009.

[23] J. H. Lv, Y. Miao, H. P. Zhu, and Y. P. Li, "A Kriging interpolationbased boundary face method for 3D potential problems," Engineering Analysis with Boundary Elements, vol. 37, no. 5, pp. 812-817, 2013.

[24] Y. T. Gu and G. R. Liu, "A boundary point interpolation method for stress analysis of solids," Computational Mechanics, vol. 28, no. 1, pp. 47-54, 2002.

[25] P. Lancaster and K. Salkauskas, "Surfaces generated by moving least squares methods," Mathematics of Computation, vol. 37, no. 155, pp. 141-158, 1981.

[26] J.-F. Wang, F.-X. Sun, and Y.-M. Cheng, "An improved interpolating element-free Galerkin method with a nonsingular weight function for two-dimensional potential problems," Chinese Physics B, vol. 21, no. 9, Article ID 090204, 2012.

[27] J. Wang, J. Wang, F. Sun, and Y. Cheng, "An interpolating boundary element-free method with nonsingular weight function for two-dimensional potential problems," International Journal of Computational Methods, vol. 10, no. 6, article no 1350043, Article ID 1350043, 2013.

[28] X. Li, "An interpolating boundary element-free method for three-dimensional potential problems," Applied Mathematical Modelling: Simulation and Computation for Engineering and Environmental Systems, vol. 39, no. 10-11, pp. 3116-3134, 2015.

[29] T. J. Hughes, J. A. Cottrell, and Y. Bazilevs, "Isogeometric analysis: CAD, finite elements, NURBS, exact geometry and mesh refinement," Computer Methods Applied Mechanics and Engineering, vol. 194, no. 39-41, pp. 4135-4195, 2005.

[30] R. N. Simpson, S. P. Bordas, J. Trevelyan, and T. Rabczuk, "A two-dimensional isogeometric boundary element method for elastostatic analysis," Computer Methods Applied Mechanics and Engineering, vol. 209, pp. 87-100, 2012.

[31] R. N. Simpson, S. P. A. Bordas, H. Lian, and J. Trevelyan, "An isogeometric boundary element method for elastostatic analysis: 2D implementation aspects," Computers \& Structures, vol. 118, pp. 2-12, 2013.

[32] M. A. Scott, R. N. Simpson, J. A. Evans et al., "Isogeometric boundary element analysis using unstructured T-splines," Computer Methods Applied Mechanics and Engineering, vol. 254, pp. 197-221, 2013.

[33] M. J. Peake, J. Trevelyan, and G. Coates, "Extended isogeometric boundary element method (XIBEM) for two-dimensional
Helmholtz problems," Computer Methods Applied Mechanics and Engineering, vol. 259, pp. 93-102, 2013.

[34] W. Zhou, B. Liu, Q. Wang et al., "NURBS-enhanced boundary element method based on independent geometry and field approximation for 2D potential problems," Engineering Analysis with Boundary Elements, vol. 83, pp. 158-166, 2017.

[35] J. Gu, J. Zhang, X. Sheng, and G. Li, "B-spline approximation in boundary face method for three-dimensional linear elasticity," Engineering Analysis with Boundary Elements, vol. 35, no. 11, pp. 1159-1167, 2011.

[36] J. Zhang, Z. Yao, and H. Li, "A hybrid boundary node method," International Journal for Numerical Methods in Engineering, vol. 53, no. 4, pp. 751-763, 2002.

[37] Y. J. Liu and N. Nishimura, "The fast multipole boundary element method for potential problems: A tutorial," Engineering Analysis with Boundary Elements, vol. 30, no. 5, pp. 371-381, 2006.

[38] Z.-H. Yao, J.-D. Xu, H.-T. Wang, and X.-P. Zheng, "Simulation of CNT composites using fast multipole bem," Journal of Marine Science and Technology, vol. 17, no. 3, pp. 194-202, 2009.

[39] Q. Wang, W. Zhou, Y. Cheng, G. Ma, and X. Chang, "Fast multipole cell-based domain integration method for treatment of volume potentials in 3D elasticity problems," Engineering Computations, vol. 34, no. 6, pp. 1849-1873, 2017.

[40] Q. Wang, W. Zhou, Y. Cheng, G. Ma, and X. Chang, "Line integration method for treatment of domain integrals in $3 \mathrm{D}$ boundary element method for potential and elasticity problems," Engineering Analysis with Boundary Elements, vol. 75, pp. $1-11,2017$

[41] Q. Wang, W. Zhou, Y. Cheng, G. Ma, X. Chang, and Q. Huang, "An adaptive cell-based domain integration method for treatment of domain integrals in 3D boundary element method for potential and elasticity problems," Acta Mechanica Solida Sinica, vol. 30, no. 1, pp. 99-111, 2017.

[42] E. Pan and A. Chengz, "Treatment of body forces in singledomain boundary integral equation method for anisotropic elasticity," Transformation of Domain Effects to the Boundary, vol. 14, p. 95, 2003. 


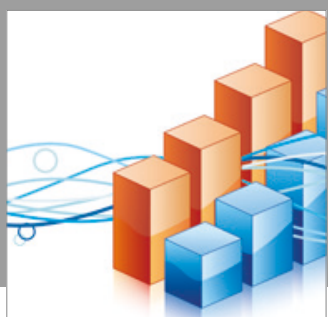

Advances in

Operations Research

vatersals

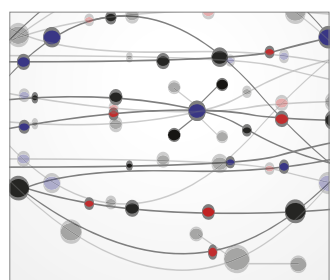

\section{The Scientific} World Journal
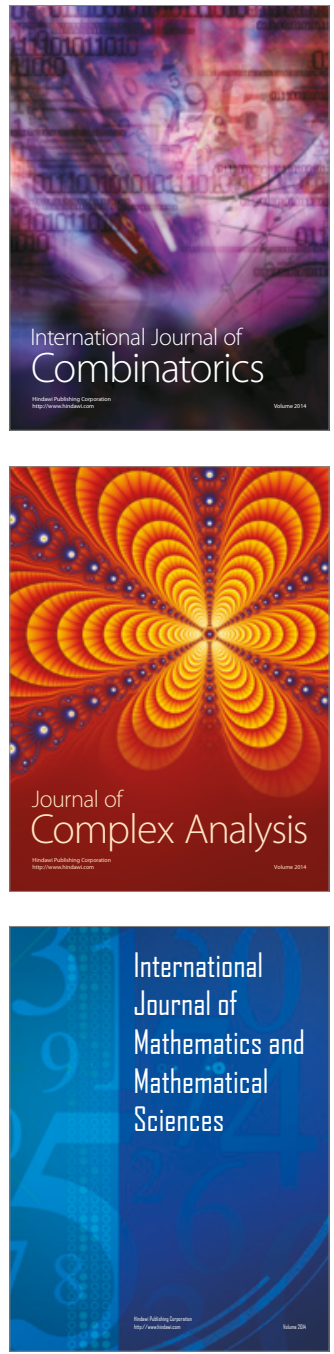
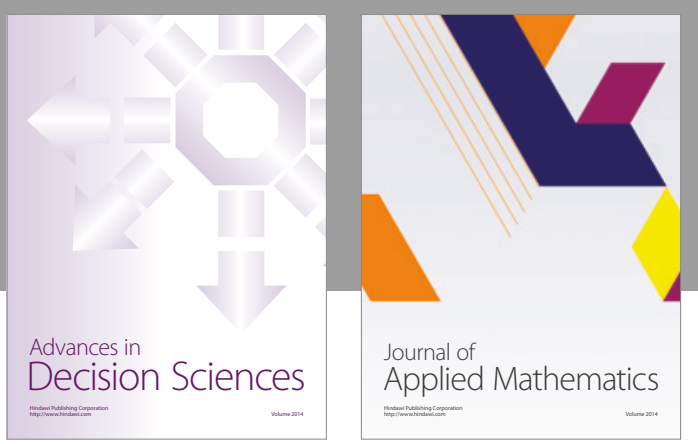

Algebra

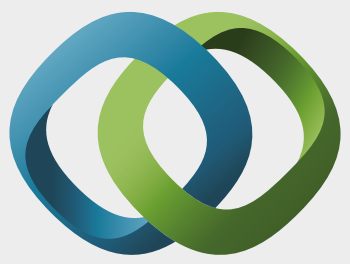

\section{Hindawi}

Submit your manuscripts at

https://www.hindawi.com
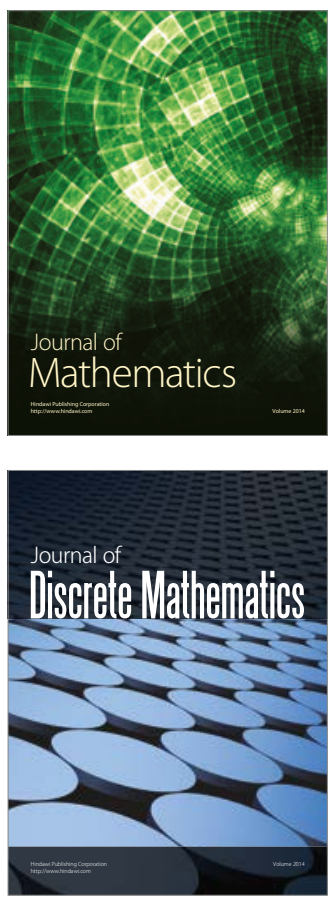

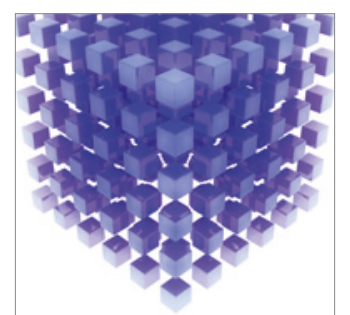

Mathematical Problems in Engineering
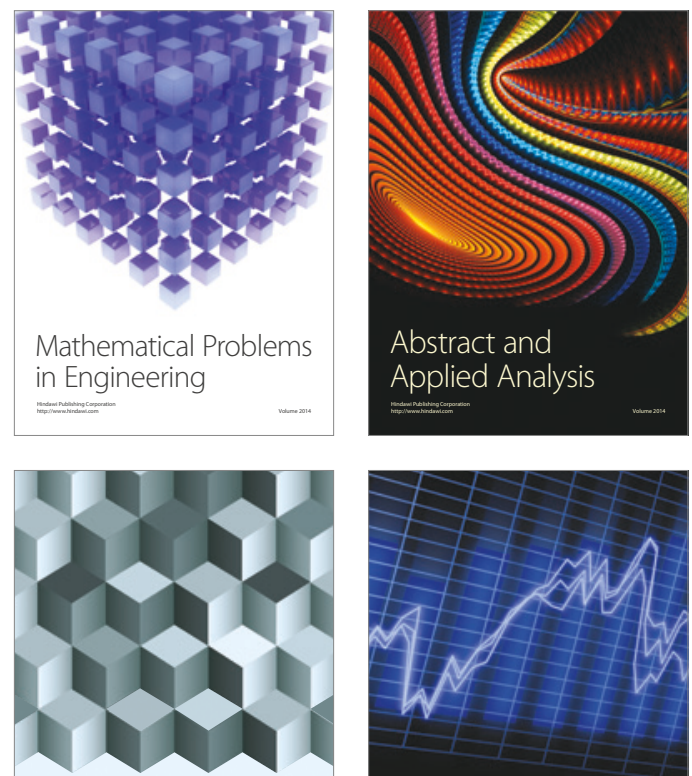

Journal of

Function Spaces

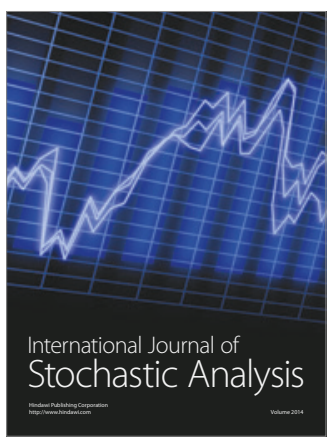

Probability and Statistics
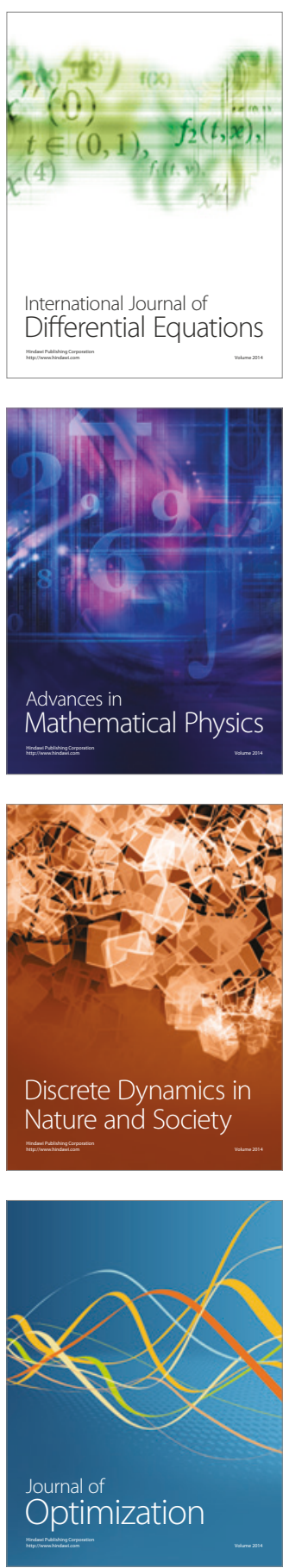